\title{
1 Intense Pulsed Electric Fields Denature Urease Proteins
}

2 Gen Urabe ${ }^{1}$, Toshiaki Katagiri ${ }^{1}$, Sunao Katsuki²

$4{ }^{1}$ Graduate School of Science and Technology, Kumamoto University 2-39-1 Kurokami, Kumamoto, 5 860-8555, Japan

6 2Institute of Pulsed Power Science, Kumamoto University 2-39-1 Kurokami, Kumamoto, 860-8555, 7 Japan

$9 \quad$ Email address: g.urabe@st.cs.kumamoto-u.ac.jp

\section{Abstract}

This paper describes the effects of nanosecond pulsed electric fields (nsPEFs) on the structure and

enzyme activity of three kinds of proteins. Intense (up to $300 \mathrm{kV} / \mathrm{cm}$ ), 5-ns-long electrical pulses were

$\mathrm{kDa}$, hexamer). We analyzed the tertiary and quaternary structures of these proteins as well as their 


\section{Text}

Electroporation (or electropermeabilization) has been widely confirmed and several attempts to investigate this phenomenon using lipid vesicles have suggested that electric pulses can damage

plasma membranes and lead to their disruption(1). Numerous studies have derived from this insight.

29 Electrochemotherapy, Ca electroporation, and tumor ablations for medical applications; non-thermal

pasteurization; and electroextraction for food-processing applications based on nanosecond pulsed

electric fields (nsPEFs) are attractive techniques and there have been many attempts to put them

into practice $(2,3)$. There are numerous accounts describing that nsPEFs lead to several biological

responses, whose kinetics have been partially obvious, such as cell-morphology transformations,

these responses have been attributed to an abrupt increase in the concentration of calcium ions,

which work as messengers to signal diverse biological reactions, triggered by the permeabilization of

the plasma membrane or the surface of the endoplasmic reticulum, promoting trans-membrane

calcium mobilization into the cytoplasm $(6,7)$.

Other cell components such as proteins, which are electrically charged dielectric compounds,

receive stress from the fields. In particular, membrane proteins are exposed to extremely high

electric fields in the order of MV/cm because the electric field is enhanced on the membrane, which is 
field. Several numerical calculations predict that proteins could respond to electricity. Microtubules

are able to transmit electrical pulses along their structures and have a dipole moment, which

generates electric fields around them(8-11). However, there are only few reports on experimentally

proven electrical effects on proteins. Amino-acid residues of a crystallized protein were found to

change their direction when exposed to a $1 \mathrm{MV} / \mathrm{cm}$ electric field(12), but because a protein crystal

was used instead of a solution, the conditions did not represent the physiological ones. Therefore, it

is important to evaluate the effects of nsPEFs on proteins under physiological conditions to

understand their physical impact and the subsequent biological reactions.

In addition, membrane proteins are under substantial electric fields in the order of $100 \mathrm{kV} / \mathrm{cm}$

because of the residual membrane potential of approximately $70 \mathrm{mV}$. It may be necessary to take

electrical perspectives into consideration to completely understand the biology. Several papers

suggest that electric fields affect the cell activity, and most of the proposed mechanisms are

supported by numerical calculations ${ }^{13-15}$.

There are several applications of studies on proteins exposed to high electric fields; controlling

intracellular electric fields may enable the manipulation of cell activities such as cell division, which

could suppress tumor growth $(15,16)$. A previous study reported that a bacterial spore wall composed

of peptidoglycan was damaged by intense pulsed electric fields of $7.5 \mathrm{kV} / \mathrm{cm}(17)$. It is critical to 
Here we focused on proteins in a liquid to simulate the intracellular conditions and analyzed

irreversible responses. Because proteins have four hierarchical structures-from primary to

quaternary-we were interested in studying the destructive effect of intense electric fields on the

primary, tertiary, and quaternary structures using several proteins with different structural features.

We have developed a 5-ns-long high-voltage pulse generator to apply intense electric fields to protein

solutions in a 1-mm-gap cuvette. Voltage pulses such as the one shown in Fig. 1, measured at the

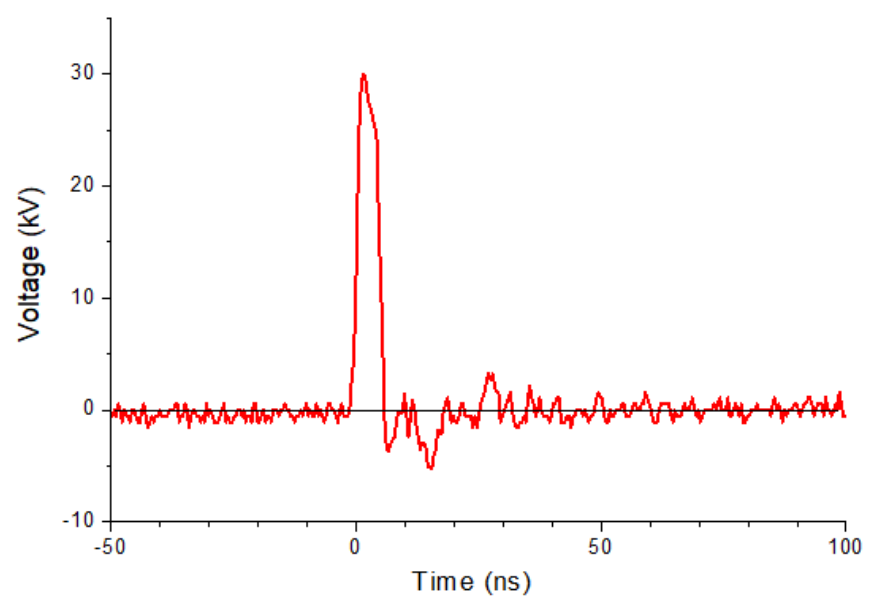

73 Figure. 1 Typical waveform of the voltage applied to the 1-mm-gap cuvette containing the protein solution.

We purchased three proteins with various structures and molar weights (all from Wako Chemicals), 
monomer), and urease from jack bean (480 kDa, hexamer), and dissolved each protein into D-PBS to

obtain $1 \mathrm{mg} / \mathrm{ml}$ protein solutions.

To analyze the effects of an electric field on the primary structure of the proteins, we exposed

81 lysozyme and albumin to nsPEFs and examined their structures using SDS-PAGE. The bands

83 the electric fields were unable to cut the peptide bonds [Figs. 2(A), (B)].

(A)

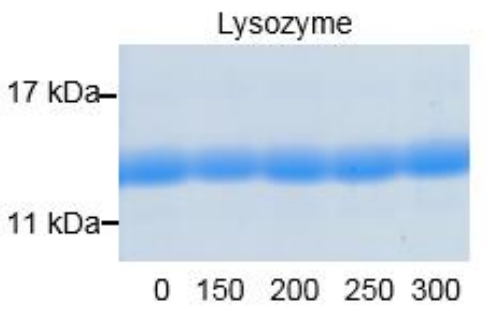

Electric Field $(\mathrm{kV} / \mathrm{cm})$

(B)

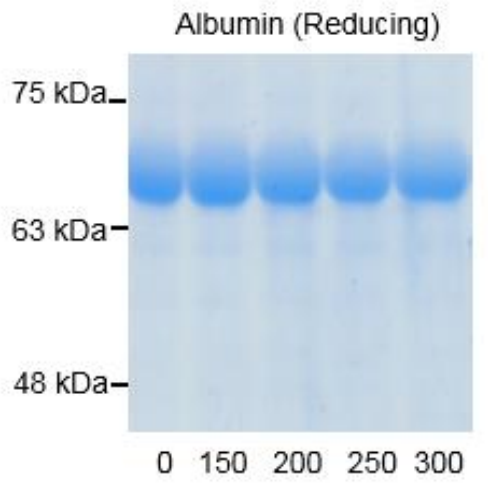

Electric Field $(\mathrm{kV} / \mathrm{cm})$

(C)

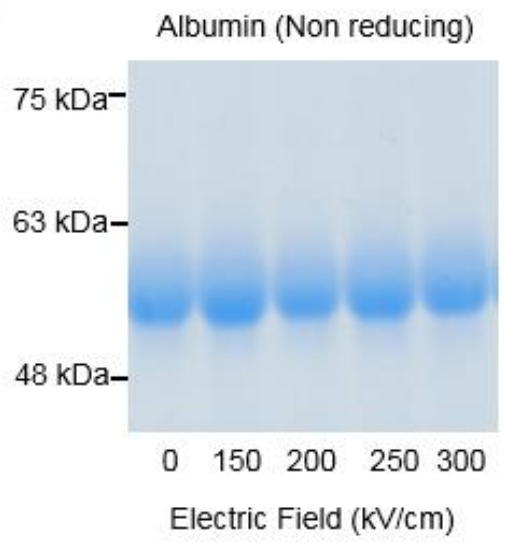

85 Figure. 2 SDS-PAGE of lysozyme and albumin exposed to nsPEFs: (A) Electrophoresis of lysozyme 
mixed with a reducing agent. The numbers below the gels represent the strength of the nsPEF. The

figures on the left side of the gels show the positions of the markers with the written molar weights.

$(\mathrm{B}, \mathrm{C})$ Electrophoresis of albumin with (B) and without (C) reducing agent.

The tertiary structures involve two binding types, namely, disulfide bonds and non-covalent bonds.

91 To evaluate the damages on the disulfide bonds, we compared albumins under two different

treatment conditions using SDS-PAGE: one of the arrangements left the disulfide bonds intact

(non-reducing conditions), whereas the other one broke them (reducing conditions). The two

conditions differed in whether 2-mercaptoethanol (2ME) (reducing agent) was added to the samples.

Albumin's band pattern did not change under reducing or under non-reducing conditions, which

implies that a $300 \mathrm{kV} / \mathrm{cm}$ nsPEF does not break the disulfide bonds [Fig. 2 (B), (C)].

Urease, which is free from covalent bonds in the tertiary and quaternary structures, was chosen as a

large "soft" protein. In the normal SDS-PAGE protocol, proteins are preheated at $95^{\circ} \mathrm{C}$ for 5 min with

SDS (surfactant) and 2ME to destroy the tertiary and secondary structures with digesting disulfide

quaternary structures. In this study, we lowered the preheating temperature and shortened the time

for 2 min was chosen to keep the bands of the trimer and subunit emerging simultaneously with the 
105 bands were visible because the hexamer is too large for the electrophoresis performed in this study

106 [Fig. 3(A)]. Under non-reducing condition, the trimer bands shifted downward and smears appeared

107 below the subunit bands when the applied electric fields were $250 \mathrm{kV} / \mathrm{cm}$ or more [Fig. 3(A), (B), (C)].

108 The trimer band also shifted under reducing conditions [Fig. 3(A), (D)], which implies that new

109 disulfide bonds could be produced between the cysteine residues in the proteins upon exposure to

$110 \mathrm{nsPEFs}(19)$. This can be a reasonable explanation for the fact that smears were observed below the

111 subunit under non-reducing conditions but disappeared under reducing conditions. The urease

112 subunits might be able to form new disulfide bonds because they contain cysteines without disulfide

113 bonds. However, the trimer bands of urease also jumped under the reducing conditions, suggesting

114 that nsPEFs not only produce disulfide bonds but also have other effects on the proteins. It has been

115 reported that electric fields can damage hydrogen bonds, thereby changing the secondary

116 structures(20). In agreement with a previous study, the urease trimer bands shifted downward under

117 both reducing and non-reducing conditions. 
bioRxiv preprint doi: https://doi org/10.1101/572784; this version posted March 11 2019. The copyright holder for this preprint (which was not certified by peer review) is the author/funder, who has granted bioRxiv a license to display the preprint in perpetuity. It is made available under aCC-BY-ND 4.0 International license.

(A)

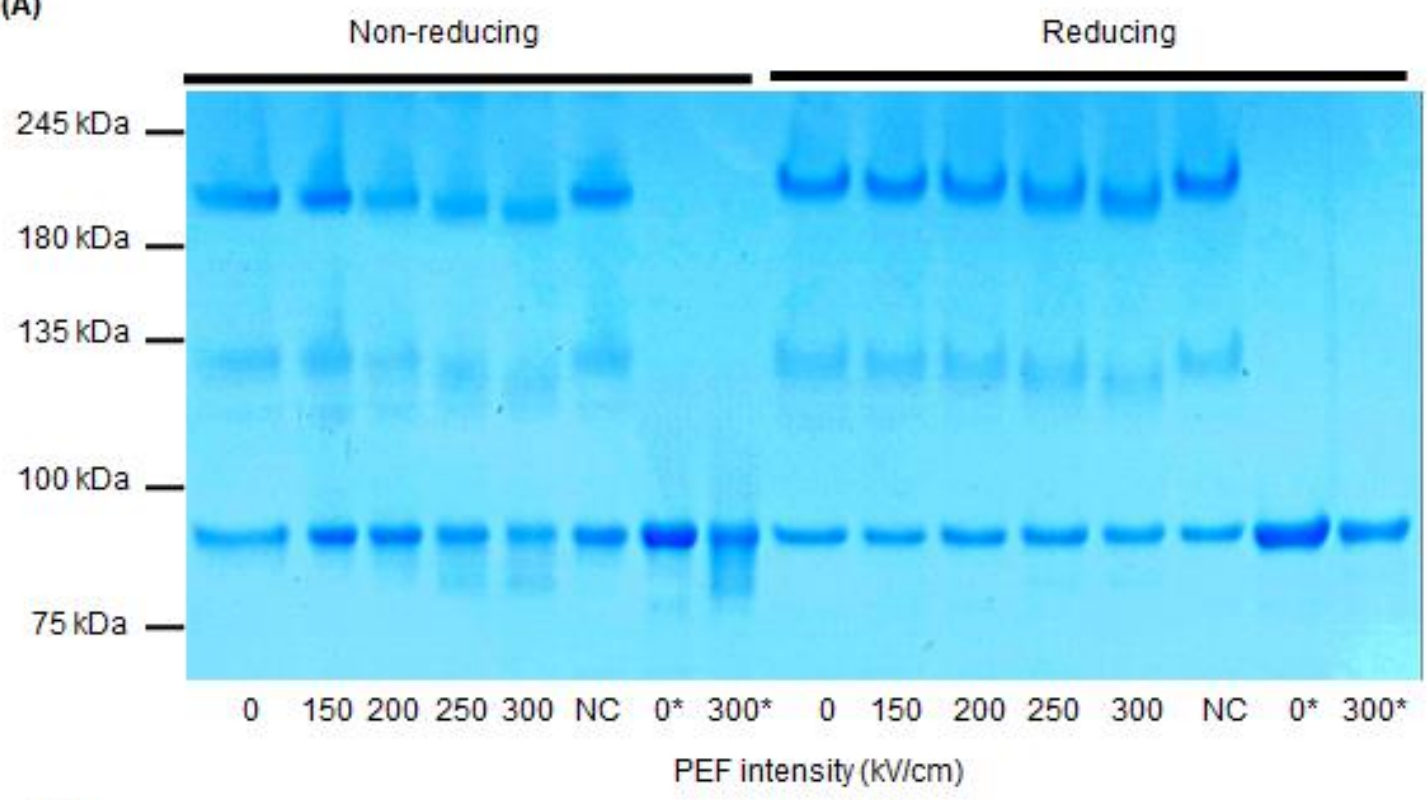

Urease trimer

(B)

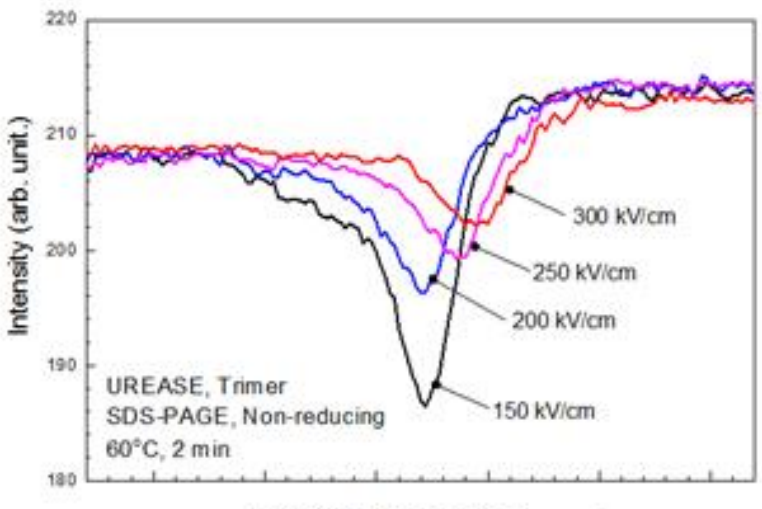

(D)

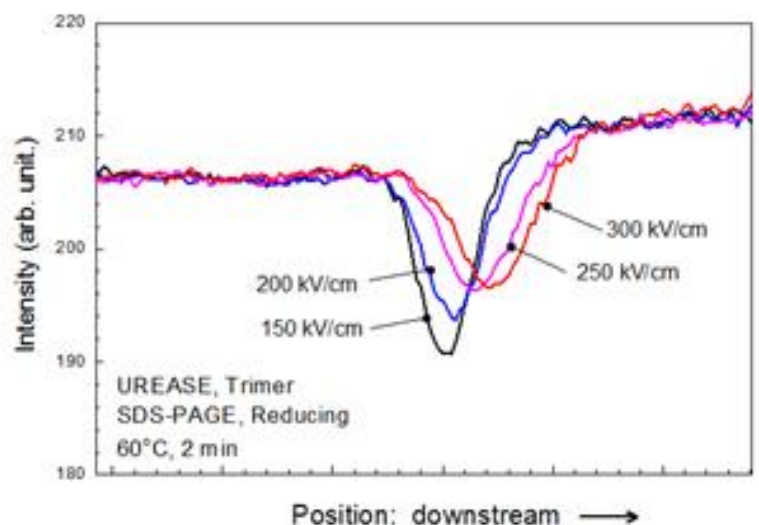

(C)

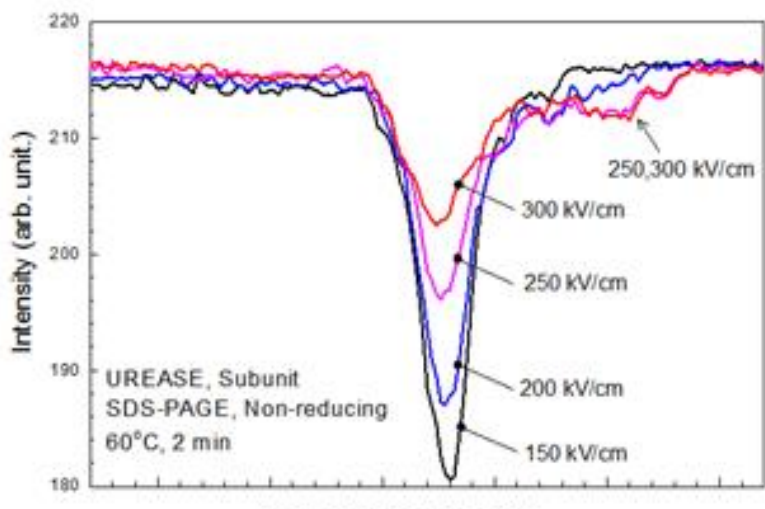

(E)

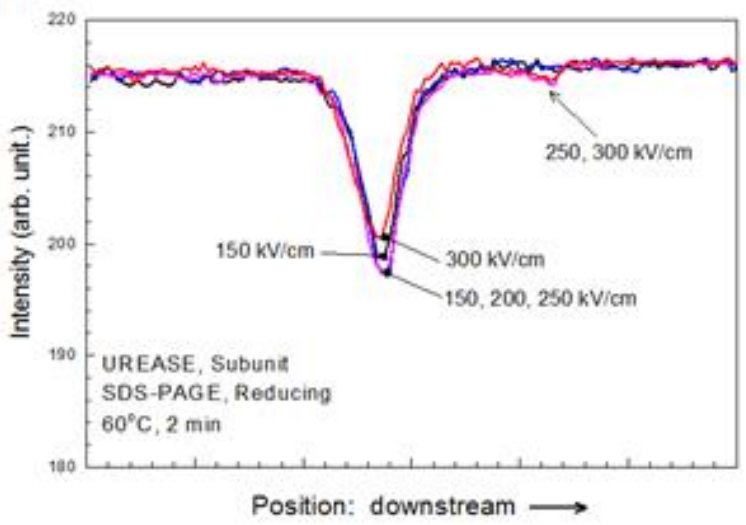

119 Figure. 3 (A) SDS-PAGE of urease treated at $60^{\circ} \mathrm{C}$ for 2 min under non-reducing or reducing

conditions. The bands between 180 and $245 \mathrm{kDa}$ correspond to the trimer whereas those between 75

and $100 \mathrm{kDa}$ correspond to the subunits. The numbers below the gel represent the strengths of the 
122 nsPEF. NC is the sample in which normal urease is dissolved in nsPEF-treated PBS. 0* and 300* are

123 the samples that were exposed to nsPEF strengths of 0 and $300 \mathrm{kV} / \mathrm{cm}$, respectively, and boiled at

$12495^{\circ} \mathrm{C}$ for 5 min. (B-E) Band-intensity distributions of SDS-PAGE: trimer bands under non-reducing

125 conditions (B), subunit bands under non-reducing conditions (C), trimer bands under reducing

126 conditions (D), and subunit bands under reducing conditions (E).

128 Although Fig. 3(A) suggests that the sum of the trimer and subunit amounts decreases with

129 increasing electric-field strength, we do not discuss the band-intensity variations because the

130 hexamer was not shown under the present SDS-PAGE conditions.

131 Protein structures are important and determine their functions. We analyzed the enzyme activity of

132 urease exposed to nsPEFs using the QuantiChrom Urease Assay Kit (BAS, DURE-100). The enzyme

133 activity is determined by the amount of ammonia (in $\mu \mathrm{mol}$ ) synthesized in $1 \mathrm{~min}$ in $1 \mathrm{~L}$ of a liquid. At

134 electric-field strengths of $250 \mathrm{kV} / \mathrm{cm}$ or more, the activity significantly decreased [Fig. 4]. This trend

135 coincides with that of the structure changes determined by SDS-PAGE [Fig. 3 (A)]. 


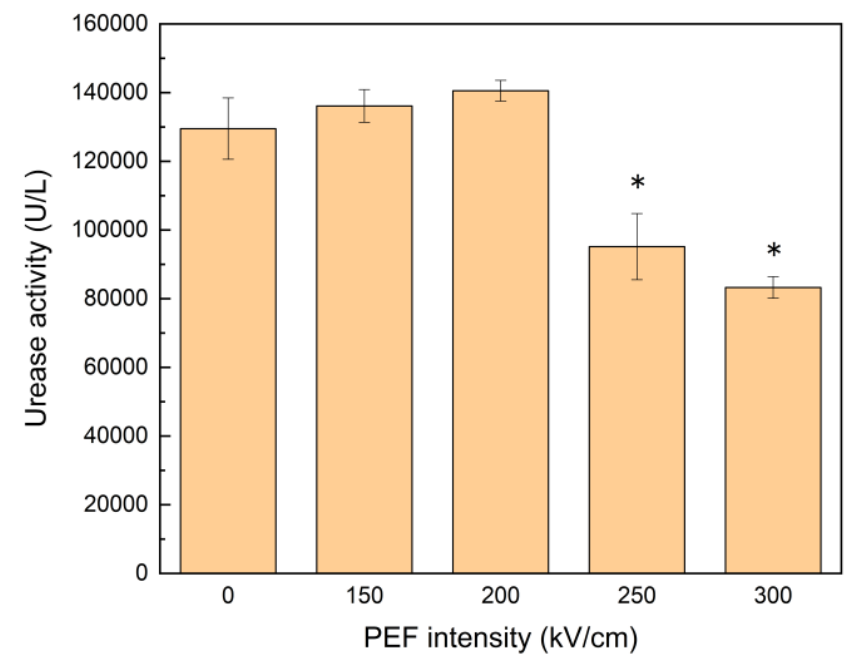

137 Figure. 4 The enzyme activity of urease exposed to nsPEFs with several electric-field strengths. The

138 error bars are described with standard error. *: $\mathrm{p}<0.01$.

In conclusion, nsPEFs with strengths above $250 \mathrm{kV} / \mathrm{cm}$ can affect not only the structure but also the

enzyme function of urease, which serves as a representative of relatively large and soft proteins,

whereas rigid proteins such as lysozyme and albumin, which have covalent bonds in their high-order

\section{Author Contributions}

146 Sunao Katsuki designed the research. Toshiaki Katagiri built and operated the pulse generator and

measured the voltage. Gen Urabe analyzed the proteins and wrote the manuscript. 
150 This study was in part supported by Grant-in-Aid for Scientific Research (17H03220).

\section{Reference}

153 1. Gianulis, E.C., J. Lee, C. Jiang, S. Xiao, B.L. Ibey, and A.G. Pakhomov. 2015. Electroporation of reversal. Sci. Rep. .

2. Bertino, G., G. Sersa, F. De Terlizzi, A. Occhini, C.C. Plaschke, A. Groselj, C. Langdon, J.J. Grau, and M. Benazzo. 2016. European Research on Electrochemotherapy in Head and Neck Cancer

3. Pérez-Andrés, J.M., C.M.G. Charoux, P.J. Cullen, and B.K. Tiwari. 2018. Chemical Modifications

(EURECA) project: Results of the treatment of skin cancer. Eur. J. Cancer. 63: 41-52.

4. Rems, L., and D. Miklavčič. 2016. Tutorial: Electroporation of cells in complex materials and tissue. 
7. Hanna, H., A. Denzi, M. Liberti, F.M. André, and L.M. Mir. 2017. Electropermeabilization of Inner and Outer Cell Membranes with Microsecond Pulsed Electric Fields: Quantitative Study with

Calcium Ions. Sci. Rep. 7: 1-14.

8. Havelka, D., M. Cifra, and O. Kučera. 2014. Multi-mode electro-mechanical vibrations of a

microtubule: In silico demonstration of electric pulse moving along a microtubule. Appl. Phys.

Lett. .

subcellular morphology. BioSystems. .

10. D. Havelka, M.C. 2009. Calculation of the Electromagnetic Field Around a Microtubule. Acta

Polytech. 49: 58-63.

11. Cifra, M., D. Havelka, O. Kučera, and J. Pokorný. 2010. Electric field generated by higher

vibration modes of microtubule. In: Proceedings of 15th Conference Microwave Techniques,

COMITE 2010. .

12. Hekstra, D.R., K.I. White, M.A. Socolich, R.W. Henning, V. Šrajer, and R. Ranganathan. 2016.

Electric-field-stimulated protein mechanics. Nature. 540: 400-405.

13. Havelka, D., M. Cifra, O. Kučera, J. Pokorný, and J. Vrba. 2011. High-frequency electric field and radiation characteristics of cellular microtubule network. J. Theor. Biol. .

14. Carr, L., S.M. Bardet, R.C. Burke, D. Arnaud-Cormos, P. Leveque, and R.P. O'Connor. 2017. 
U87 human glioblastoma cells. Sci. Rep. .

189 15. Zhao, Y., and Q. Zhan. 2012. Electric fields generated by synchronized oscillations of microtubules,

Model. .

16. Pokorný, J., J. Pokorný, and J. Kobilková. 2013. Postulates on electromagnetic activity in biological

systems and cancer. Integr. Biol. (United Kingdom). .

17. Pillet, F., C. Formosa-Dague, H. Baaziz, E. Dague, and M.P. Rols. 2016. Cell wall as a target for bacteria inactivation by pulsed electric fields. Sci. Rep. 6 .

18. Adachi, R., S. Hatayama, N. Ohnishi, S. Katsuki, T. Wada, and K. Abe. 2018. Cell Death Induced by Nanosecond Pulsed Electric Fields and its Dependence on Pulse Duration. Electron. Commun.

Japan. 101: 38-48.

19. Zhao, W., Y. Tang, L. Lu, X. Chen, and C. Li. 2014. Review: Pulsed Electric Fields Processing of

Protein-Based Foods. Food Bioprocess Technol. 7: 114-125.

20. Bekard, I., and D.E. Dunstan. 2014. Electric field induced changes in protein conformation. Soft

Matter. 10: 431-437. 